Research Paper

\title{
Plasma mRNA as liquid biopsy predicts chemo- sensitivity in advanced gastric cancer patients
}

\author{
Jie Shen ${ }^{*}$, Weiwei Kong ${ }^{1 *}$, Yuanna Wu1 ${ }^{1}$, Haozhen Ren ${ }^{2}$, Jia Wei1, Yang Yang1, Yan Yang1, Lixia Yu1, \\ Wenxian Guan², Baorui Liu ${ }^{\boxplus 凶}$ \\ 1. The Comprehensive Cancer Centre of Drum Tower Hospital, Medical School of Nanjing University, Clinical Cancer Institute of Nanjing University, 321 Zhongshan Rd, \\ Nanjing 210008, China; \\ 2. Department of Surgery, Drum Tower Hospital, Medical School of Nanjing University, 321 Zhongshan Rd, Nanjing 210008, China. \\ *Jie Shen, Weiwei Kong contributed equally to this work. \\ $\triangle$ Corresponding authors: Baorui Liu, MD, PhD, The Comprehensive Cancer Centre of Drum Tower Hospital, Medical School of Nanjing University, Clinical Cancer \\ Institute of Naniing University, 321 Zhongshan Rd, Nanjing 210008, China. Fax: +86-25-83105082, Email: baoruiliu@nju.edu.cn and Wenxian Guan, MD, PhD, Department of \\ General Surgery, Drum Tower Hospital, Medical School of Nanjing University, 321 Zhongshan Rd, Nanjing 210008, China. E-mail: uanwenxiannj@163.com. \\ (c) Ivyspring International Publisher. This is an open access article distributed under the terms of the Creative Commons Attribution (CC BY-NC) license \\ (https://creativecommons.org/licenses/by-nc/4.0/). See http://ivyspring.com/terms for full terms and conditions.
}

Received: 2016.08.28; Accepted: 2016.11.14; Published: 2017.02.10

\begin{abstract}
Predictive biomarkers based individualized chemotherapy can improve efficacy. However, for those advanced patients, it may be impossible to obtain the tissues from operation. Tissues from biopsy may not be always enough for gene detection. Thus, biomarker from blood could be a non-invasive and useful tool to provide real-time information in the procedure of treatment. To further understand the role of plasma mRNA in chemo-efficiency prediction, several mRNA expression levels were assessed in plasma and paired tumor tissues from 133 locally advanced gastric cancer patients (stage III), and mRNA levels were correlated with chemosensitivity to docetaxel, pemetrexed, platinum, and irinotecan. mRNA expression level in 64 advanced gastric cancer patients (stage IV) was also examined (55 in test group, and 9 in control), and chemotherapy in the test group were given according to the plasma gene detection. As a result, in the 133 patients with locally advanced gastric cancer (Stage III), correlations were observed between the mRNA expression of plasma/tumor BRCA1 levels and docetaxel sensitivity $(P<0.001)$, plasma/tumor TS and pemetrexed sensitivity $(P<0.001)$, plasma/tumor $B R C A 1$ and platinum sensitivity ( $\mathrm{Plasma}, \mathrm{P}=0.016$; tumor, $\mathrm{P}<0.001$ ), and plasma/tumor TOPOI and irinotecan sensitivity (plasma, $\mathrm{P}=0.015$; tumor, $\mathrm{P}=0.011$ ). Among another 64 patients with advanced cancer (Stage IV), the median OS of test group was $15.5 \mathrm{~m}(95 \% \mathrm{Cl}=10.1$ to $20.9 \mathrm{~m})$, the PFS was $9.1 \mathrm{~m}(95 \% \mathrm{Cl}=8.0$ to $10.2 \mathrm{~m})$, which were significant longer than the control $(\mathrm{P}=0.047$ for $\mathrm{OS}, \mathrm{P}=0.038$ for PFS). The mortality risk was higher in the control than patients treated according to the plasma gene detection (HR in the control $=2.34,95 \% \mathrm{Cl}=0.93$ to $5.88, \mathrm{P}=0.071$ ). Plasma $\mathrm{mRNA}$ as liquid biopsy could be ideal recourse for examination to predict chemo-sensitivity in gastric cancer.
\end{abstract}

Key words: Plasma mRNA; liquid biopsy; gastric cancer; chemosensitivity; individualized chemotherapy.

\section{Introduction}

The routine chemotherapy for the first-line treatment of advanced gastric cancer tends to include a platinum-fluoropyrimidine doublet [1]; however, the median survival time remains around one year. No standard treatment method was recommended for second-line therapy, although some agents such as docetaxel and irinotecan have shown some activity [2]. Studies have demonstrated that predictive biomarkers based individualized chemotherapy can improve efficacy. For example, studies have shown that BRCA1 (breast cancer susceptibility gene 1) mRNA expression level is correlated with sensitivity of cisplatin and docetaxel [3,4]. We have also demonstrated in our previous work that mRNA expression level of BRCA1 was correlated with tumor sensitivity to platinum negatively, but associated with sensitivity of docetaxel positively in gastric cancer patients $[5,6]$. 
Up to now, tumor itself is the basic resource for the examination of biomarkers. Undeniable, there were some shortages of tumor tissue based detection. Firstly, for those advanced patients, it may be impossible to obtain the tissues from operation. Tissues from biopsy may not be always enough for gene detection. Secondly, there would be some disaccord if using tissue from initial diagnosis to reflect gene expression alterations when the time of clinical progression [7], considering primary and metastatic tumors can be different at transcriptomic and genomic levels even from the same patient [7-11]. Moreover, gene expression levels may change after chemotherapy $[12,13]$. In such case, cell-free tumor-related nucleic acids can be a non-invasive and useful tool for tracking changes $[8,14]$, providing useful information to help determining the optimal treatment, and can be helpful for those who did not have enough tissues for gene detection when neoadjuvant or adjuvant chemotherapy needed to be chosen.

In our previous work, by establishing a practical method for plasma mRNA detection, we have demonstrated that plasma BRCA1 and TS (Thymidylate Synthase) mRNA levels were correlated to the sensitivity of docetaxel and pemetrexed respectively in gastric cancer $[15,16]$. However, that is only a preclinical study and the chemo-sensitivity was tested in vitro. Moreover, although it also has been demonstrated that mRNA expression level of ERCC1 (Excision Repair Cross Complementation Group1), TOPO1(Topoisomerase1) and APTX (Aprataxin) in tumor tissues has relationship with platitum and irinotecan sensitivity in our previous work[17-19], the role of plasma expression of those genes in gastric cancer therapy is still unclear. Therefore, in the current study, we try to identify if plasma mRNA levels of those genes can be an ideal recourse for examination in chemo-sensitivity prediction, firstly in a pilot study of 133 patients with locally advanced gastric cancer (Stage III) and then in a validation set of 64 patients with advanced gastric cancer (Stage IV).

\section{Materials and Methods}

\section{Study design and patients}

Figure 1 shows the experiments performed. In the pilot study, 133 paired samples of fresh tumor tissues and blood were collected in the Comprehensive cancer center of Drum Tower Hospital, from the year of 2010 to 2012. The fresh tumor tissues and paired blood samples were sent at the condition of $4^{\circ} \mathrm{C}$ to the gene testing center for examination within 15-20 minutes once collected. All of patients were histologically proven locally advanced (stage III).

In the validation study, another 64 patients with stage IV gastric cancer were admitted from November 2013 to November 2014. All the patients were histologically proven metastatic gastric cancer and chemo or radiation naïve with ECOG PS (Eastern Cooperative Oncology Group, performance status) $\leq$ 2. Among them 55 patients were the test group and 9 were the control. In the test group, chemotherapy was given according to the plasma gene detection, while the control was given the chemotherapy of docetaxel plus S-1. Those 64 patients were further followed up. Their overall survival time (OS) was calculated from the date of diagnosis to the date of last follow-up or death from any cause, and the progress free survival (PFS) was from the date of diagnosis to the date of progress. The study has agreement from the Drum Tower Hospital' Institutional Ethics Review Board. The informed consents were signed by all the patients. Investigators were blinded to knowledge of the samples.

\section{Pilot study}

The pilot study was carried out in 133 gastric cancer patients (stage III). Sensitivity to agents, including docetaxel, pemetrexed, platinum and irinotecan was examined in vitro for every tumor sample [15-18]. In addition, each tumor sample was also formalin fixed, paraffin embedded (FFPE), and then tested for all the genes mRNA levels, including BRCA1, ERCC1, TOPO1, TS, and APTX. All of those gene expressions were tested in the blood samples as well.

\section{Validation study}

In view of the pilot study, further work was undertaken to investigate the findings and identify the potential chemo-efficiency prediction value of plasma biomarkers in a cohort of 64 stage IV gastric cancer patients. All those patients were chemo or radiation naïve and planned to receive the first line chemotherapy. The mRNA levels in plasma which have been approved to be useful in the pilot study were assessed and chemotherapy was given accordingly (Figure 2). Chemo schedule and dose for different strategy were as follows:

1) Docetaxel + S-1: Docetaxel $35-45 \mathrm{mg} / \mathrm{m}^{2}$, VD, d1, 15, S-1 40mg/m², bid, po, d1-14, q28d.

2) Platinum + S-1: Oxaliplatin $85 \mathrm{mg} / \mathrm{m}^{2}, \mathrm{VD}, \mathrm{d} 1$, 15, S-1 40mg/m², bid, po, d1-14, q28d.

3) Pemetrxed + S-1: Pemetrxed 500mg/m2, VD, d1, S-1 40mg/m², bid, po, d1-14, q21d.

4) Irinotecan + S-1: Irinotecan $180 \mathrm{mg} / \mathrm{m}^{2}, \mathrm{~d} 1,15$, S-1 40mg/m², bid, po, d1-14, q28d. 


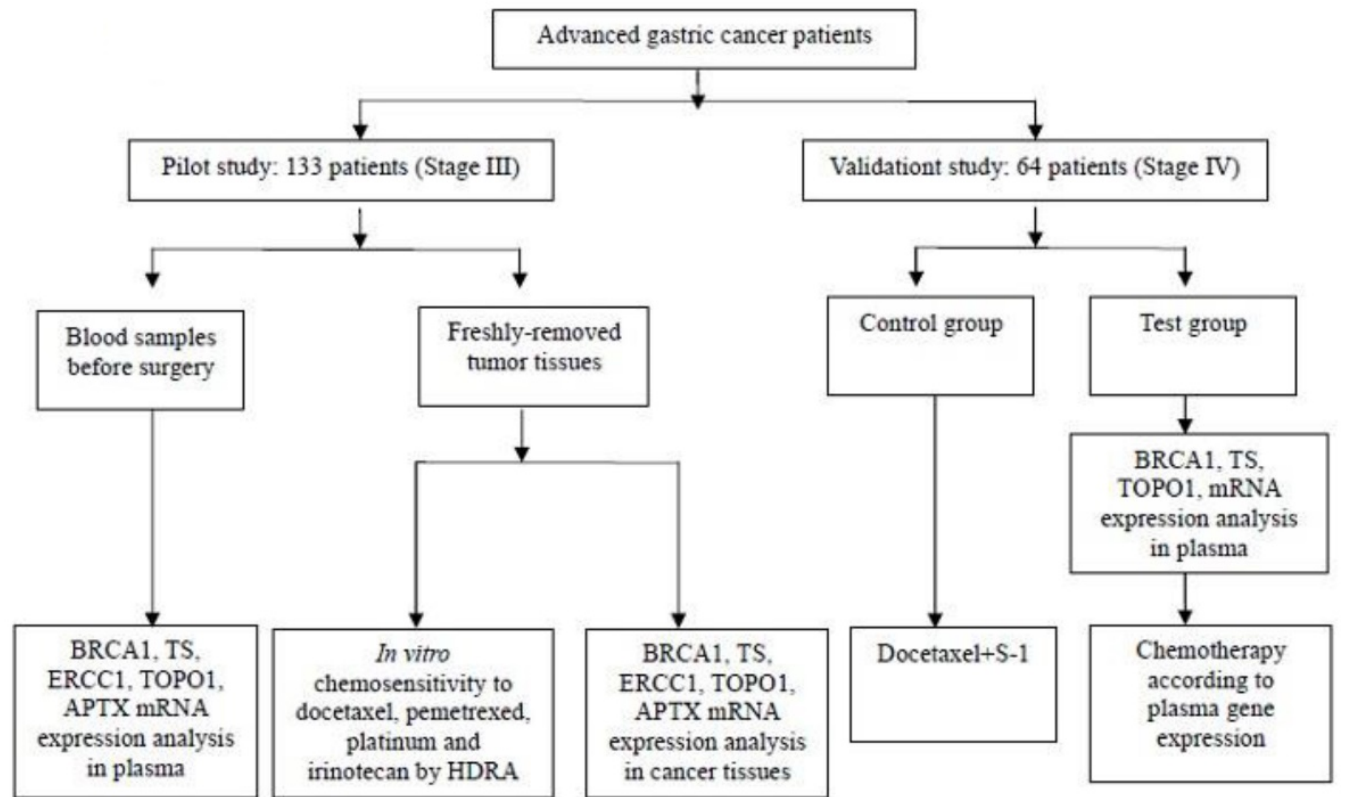

Figure 1. Flow Chart Showing Patient Disposition and Experiments Performed.

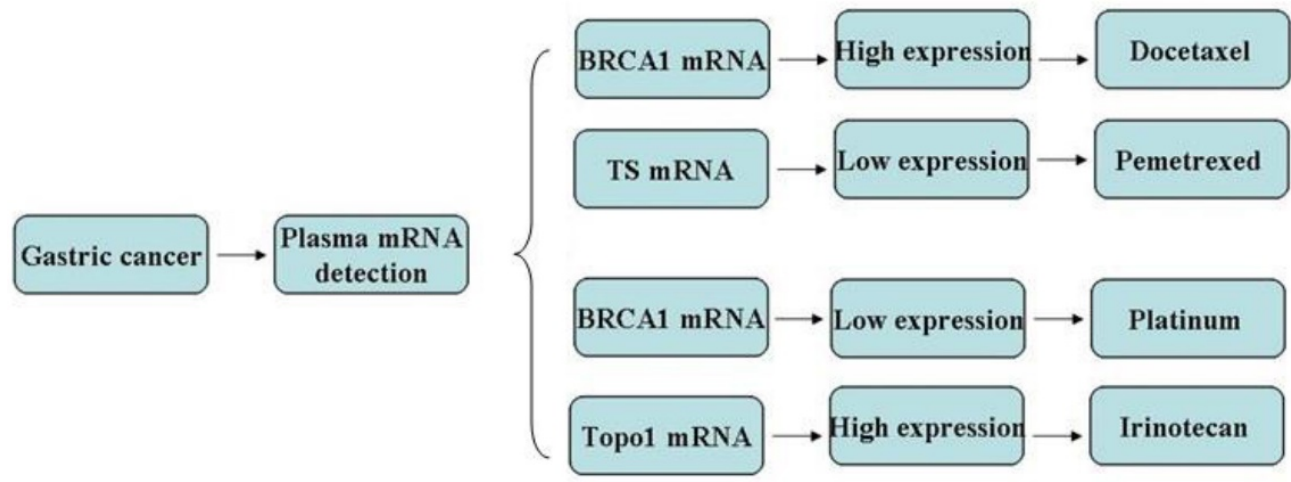

Figure 2. Chemotherapy regency were given according to the gene expression in the test group.

\section{mRNA expression in the pilot and validation studies}

The method for mRNA expression detection in plasma and tumor were reported previously by our team [15-18]. Briefly speaking, blood from gastric cancer patients were collected, and sent to the laboratory at the condition of $4^{\circ} \mathrm{C}$ as soon as possible, within 15-20 min. And then plasma samples' and FFPE tissues' total RNA were extracted, purified and reverse transcribed as reported in our previous work[15-18]. Template cDNAs were amplified with specific primers and probes purchased from Applied Biosystems (USA). The condition for PCR was $50^{\circ} \mathrm{C}$ for 2 mins, $95^{\circ} \mathrm{C}$ for 15 mins, 40 cycles at $95^{\circ} \mathrm{C}$ for $15 \mathrm{~s}$, and followed by $60^{\circ} \mathrm{C}$ for $1 \mathrm{~min}$. The primers and probes were as follows:

BRCA1(NM_007294), forward: 5'GGCTA TCCTC TCAGA GTGAC ATTTTA 3',

reverse: 5'GCTTT ATCAG GTTAT GTTGC ATGGT 3', probe: 6FAM -5'CCACT CAGCA GAGGG 3' MGB;

TS Assay IDs: Hs00426586_m1; ERCC1 Assay IDs: Hs00157415_m1; TOPO1 Assay IDs: Hs00243257_m1; APTX Assay IDs: Hs00214452_m1*;

ACTB (used as endogenous control, NM_001101.3), forward: 5’ TGAGC GCGGC TACAG CTT 3', reverse: 5' TCCTT AATGT CACGC ACGAT TT 3', probe: 6FAM -5'ACCAC CACGG CCGAG CGG 3'TAMRA.

Relative level of gene expression quantification was calculated using the formula of comparative $\mathrm{Ct}$ method [15-18].

\section{Statistical analyses}

The Mann-Whitney $U$ tests were adopted to compare chemo-sensitive and chemo-resistant samples based on gene expression level in tumor and plasma. The Kruskal-Wallis test and the Mann-Whitney $U$ test were adopted to analyze relationship between sensitivity of each 
chemotherapeutic agent and clinical characteristic, and also between each mRNA level and clinical characteristic. Survival curves were obtained by the Kaplan-Meier method, and survival differences assessed using the Logrank test. The Cox proportional hazards model was used to assess the simultaneous contribution on overall survival of different covariates. Statistical significances were set at $\mathrm{P}<0.05$. Statistical analysis was finished by using SPSS, version 19.0 .

\section{Results}

Characteristics of patients were shown in Table 1 (Pilot study: preclinical study with patients of stage III) and Table 4 (Validation study: clinical study with patients of stage IV). In the pilot study, the majority of patients were males $(75.9 \%)$, and the histology was adenocarcinoma in all the patients. In 51 (38.3\%) patients, the tumor was located in the distal stomach, in $52(39.1 \%)$ in the proximal stomach, and in 30 $(22.6 \%)$ in the whole stomach. All patients had stage III disease, and lymph node metastases were present in all the patients.

Table 1. Patient Characteristics of pilot study

\begin{tabular}{ll}
\hline Characteristic & Validation Study N=133 \\
\hline Age, y median (range) & $60(31-82)$ \\
$<60$ y & 59 \\
$>=60 y$ & 74 \\
Sex & \\
Male & 101 \\
Female & 32 \\
Tumor Site & \\
Distal stomach & 51 \\
Proximal stomach & 52 \\
Whole stomach & 30 \\
Stage & \\
III & 133 \\
Histological grade & \\
2 & 27 \\
3 & 67 \\
Mixed 2-3 & 39 \\
Lymph node metastasis & \\
Yes & 133 \\
\hline
\end{tabular}

BRCA1, TS, ERCC1, TOPO1, and APTX mRNA expression was successfully tested in the paired plasma and tissues from 133 patients. The mean and range of plasma and tumor mRNA expression levels of each gene were shown in Table 2. We did not find any significant association between plasma/tumor mRNA levels or in vitro chemosensitivity with clinical characteristics (data not shown). However, correlations were observed between the mRNA expression levels of plasma/tumor BRCA1 levels and docetaxel sensitivity $(\mathrm{P}<0.001)$, plasma/tumor TS and pemetrexed sensitivity $(\mathrm{P}<0.001)$, plasma/tumor
BRCA1 and platinum sensitivity (plasma, $\mathrm{P}=0.016$; tumor, $\mathrm{P}<0.001$ ), and plasma/tumor TOPO1 and irinotecan sensitivity (plasma, $\mathrm{P}=0.015$; tumor, $\mathrm{P}=0.011$, Figure 3A-D). There no correlation between ERCC1 or APTX with chemosensitivity. According to the pilot study, we further chose BRCA1, TS and TOPO1 for validation study. The cut-offs for high and low expression levels were as Table 3 .

Table 2. Mean mRNA levels in plasma and tumor

\begin{tabular}{llll}
\hline Gene & N & \multicolumn{2}{c}{ Mean mRNA values (range) } \\
\hline & & Plasma & Tumor \\
\hline BRCA1 & 133 & $1.10(0.06-3.77)$ & $7.80(0.24-30.80)$ \\
TS & 133 & $1.23(0.02-7.25)$ & $9.78(0.13-46.55)$ \\
ERCC1 & 133 & $0.31(0.02-1.80)$ & $15.00(0.33-46.30)$ \\
TOPO1 & 133 & $0.68(0.01-4.36)$ & $7.60(0.56-37.81)$ \\
APTX & 133 & $0.45(0.01-2.17)$ & $4.30(0.26-17.99)$ \\
\hline
\end{tabular}

Table 3. The Cut-off for plasma gene expression

\begin{tabular}{llll}
\hline & BRCA1 & TOPO1 & TS \\
\hline Low expressed & BRCA1 $<0.755$ & TOPO1 $<0.376$ & TS $<0.604$ \\
Media expressed & $0.755 \leq$ & $0.376 \leq$ TOPO1 $<0.839$ & $0.604 \leq$ TS $<0.873$ \\
& BRCA1<2.163 & & \\
High expressed & $2.163 \leq$ BRCA1 & $0.839 \leq$ TOPO1 & $0.873 \leq \mathrm{TS}$ \\
\hline
\end{tabular}

Table 4. Patient characteristics of 64 stage IV gastric cancer patients.

\begin{tabular}{|c|c|c|c|}
\hline Characteristic & $\begin{array}{l}\text { Totol N=64 } \\
N(\%)\end{array}$ & $\begin{array}{l}\text { Test } N=55 \\
N(\%)\end{array}$ & $\begin{array}{l}\text { Control } \\
N=9 \mathrm{~N}(\%)\end{array}$ \\
\hline Age, y median (range) & $62(35-79)$ & $62(35-79)$ & $63(48-77)$ \\
\hline$<60 y$ & 25 & 22 & 3 \\
\hline$>=60 y$ & 39 & 33 & 6 \\
\hline \multicolumn{4}{|l|}{ Sex } \\
\hline Male & 47 & 39 & 8 \\
\hline Female & 17 & 16 & 1 \\
\hline \multicolumn{4}{|l|}{ Lauren } \\
\hline Intestinal type & 17 & 15 & 2 \\
\hline Diffuse type & 7 & 5 & 2 \\
\hline Mixed type & 5 & 5 & 0 \\
\hline Unknown & 35 & 30 & 5 \\
\hline \multicolumn{4}{|l|}{ Histological grade } \\
\hline 1 & 0 & 0 & 0 \\
\hline 2 & 34 & 28 & 6 \\
\hline 3 & 16 & 14 & 2 \\
\hline Unknown & 14 & 13 & 1 \\
\hline \multicolumn{4}{|l|}{ Palliative operation } \\
\hline No & 47 & 40 & 7 \\
\hline Yes & 17 & 15 & 2 \\
\hline \multicolumn{4}{|l|}{ Metastasis site } \\
\hline Liver & 28 & 24 & 4 \\
\hline Lung & 7 & 7 & 0 \\
\hline Pancreas & 3 & 1 & 2 \\
\hline Adrenal gland & 2 & 2 & 0 \\
\hline $\begin{array}{l}\text { Retroperitoneal, or longitudinal, or } \\
\text { supraclavicular lymph nodes }\end{array}$ & 22 & 19 & 3 \\
\hline Malignant pleural effusion or ascites & 3 & 3 & 0 \\
\hline Abdominal cavity seeding & 18 & 15 & 3 \\
\hline Bone & 7 & 7 & 0 \\
\hline \multicolumn{4}{|l|}{ Chemotherapy } \\
\hline Docetaxel+S-1(BRCA1 $1^{\text {high }}$ ) & 22 & 13 & 9 \\
\hline Oxaliplatin+S-1( BRCA1 low) & 21 & 21 & 0 \\
\hline Pemetrexed+S-1(TSlow) & 14 & 14 & 0 \\
\hline Irinotecan+S-1(TOPO1 high) & 7 & 7 & 0 \\
\hline
\end{tabular}



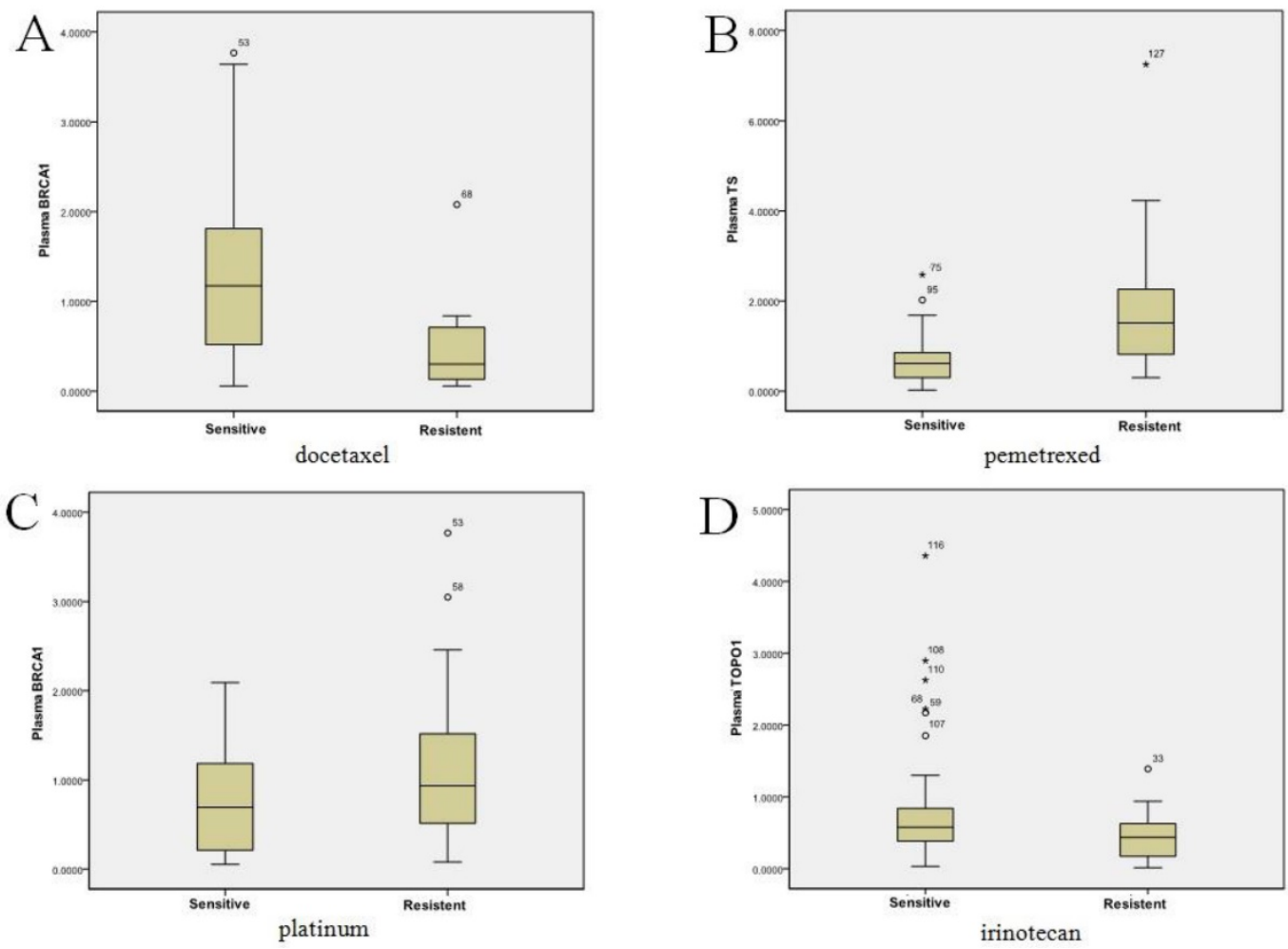

Figure 3. Chemosensitivity in vitro was associated with the plasma mRNA expression levels of BRCA1, TS and TOPOI in 133 gastric cancer patients. Figure $3 A$ : plasma BRCA1 levels and docetaxel sensitivity $(\mathrm{P}<0.001)$; Figure 3B: plasma TS levels and pemetrexed sensitivity $(\mathrm{P}<0.001)$; Figure $3 \mathrm{C}$ : plasma BRCA1 levels and platinum sensitivity $(P=0.016)$; Figure 3D: plasma TOPOI levels and irinotecan sensitivity $(P=0.015)$.

In the validation study, characteristics of patients were shown in Table 4 (clinical study with patients of stage IV). The majority of patients were males (73.4\%), and the histology was adenocarcinoma in all the patients. Majority of the patients did not receive palliative operation. In $50(78.1 \%)$ patients, the tumor was histological grade 2 and 3, while the rest patients were unknown. All patients had stage IV disease, with metastasis sites including liver, lung, pancreas, adrenal gland, retroperitoneal, or longitudinal, or supraclavicular lymph nodes, malignant pleural effusion or ascites, abdominal cavity seeding and bone. 9 patients were treated with docetaxel plus S-1 as control (none of those patients were with BRCA1 mRNA high expression). 13 patients with higher expressed plasma BRCA1 were treated with docetaxel plus S-1, 21 patients with lower expressed plasma BRCA1 were treated with oxaliplatin plus S-1, 14 patients with lower expressed plasma TS were treated with pemetrexed plus S-1, and 7 patients with higher expressed plasma TOPO1 were treated with irinotecan plus S-1.

All patients were diagnosed as stage IV when admitted to this study, of which 55 patients were treated according to their gene detection (Table 4), and another 9 were treated with docetaxel+S-1 as control. With the median follow-up of $14.0 \mathrm{~m}$ (range, 2.7-35.2m), Kaplan-Meier survival analysis showed that median OS was $14.3 \mathrm{~m}$ ( $95 \%$ CI [confidence interval $]=12.5$ to $16.2 \mathrm{~m}$ ) for all the 64 patients. The median OS for 55 patients receiving individualized chemotherapy according to plasma gene detection was $15.5 \mathrm{~m}(95 \% \mathrm{CI}=10.1$ to $20.9 \mathrm{~m})$, and PFS was $9.1 \mathrm{~m}$ (95\% CI $=8.0$ to $10.2 \mathrm{~m}$ ). In the control group, median OS was $9.9 \mathrm{~m}$ (95\% CI $=3.2$ to $16.5 \mathrm{~m})$, and PFS was $6.2 \mathrm{~m}(95 \% \mathrm{CI}=0$ to $12.9 \mathrm{~m})$, showing statistically significant differences in PFS $(P=0.038)$ and OS $(P=$ 0.047 , Figure $4 \mathrm{~A}-\mathrm{B})$. The 1-year survival rate was $64.7 \%$ of patients treated according to plasma gene detection and $33.3 \%$ in the control. The 2-year survival rate was $13.7 \%$ of patients treated according to plasma gene detection and $0 \%$ in the control. There were no significant differences of PFS (Table 5, Figure 5A) or OS (Table 6, Figure 5B) between different groups of individualized chemotherapy except group oxalipaltin +S-1 and docetaxel +S-1.

Table 5. PFS between different groups of individualized chemotherapy.

\begin{tabular}{llll}
\hline P & Oxalipatin+S-1 & Pemetrexed+S-1 & Irinotecan+S-1 \\
\hline Docetaxel+S-1 & 0.016 & 0.441 & 0.459 \\
Oxalipatin+S-1 & & 0.162 & 0.258 \\
Pemetrexed+S-1 & & & 0.397 \\
\hline
\end{tabular}


Table 6. OS between different groups of individualized chemotherapy.

\begin{tabular}{llll}
\hline $\mathbf{P}$ & Oxalipatin+S-1 & Pemetrexed+S-1 & Irinotecan+S-1 \\
\hline Docetaxel+S-1 & 0.035 & 0.435 & 0.334 \\
Oxalipatin+S-1 & & 0.179 & 0.201 \\
Pemetrexed+S-1 & & & 0.504 \\
\hline
\end{tabular}

To get further insight of the role of plasma gene detection, multivariable analyses of survival time in all the patients were carried out. Patients with more than two metastasis sites had higher mortality risk compared with those with one (HR [hazard ratio] of death $=2.16,95 \% \mathrm{CI}=1.01$ to $4.65, P=0.048)$. Also, Higher mortality risk was observed in those patients with ECOG PS 2 compared with ECOG PS $<2$ (HR of death $=2.55,95 \% \mathrm{CI}=1.12$ to $5.83, P=0.026)$. Moreover, there is a trend that patients receiving doxetal+S-1 without gene detection have higher mortality compared with those receiving individualized chemotherapy based on plasma gene detection (HR of death $=2.34,95 \% \mathrm{CI}=0.93$ to $5.88, P$ $=0.071$ ) (Table 7).

As shown in Figure 6, this is one of the patients who get survival benefit from the individualized chemotherapy based on plasma gene detection. A 59-year-old man was diagnosed as low differentiation adenocarcinoma of stomach by upper-gastrointestinal (GI) endoscopy on $11^{\text {th }}$ march, 2013, during which an irregular ulcera with the length of $2.7^{*} 3.1 \mathrm{~cm}$ at the cardiac wall was noted. As the same time, more than one liver metastasis were confirmed by both type-B ultrasonic exam and CT scan with the biggest one $9.6 \mathrm{~cm}$ in diameters. Examination of a biopsy specimen led to the diagnosis, and HER2 immunohistochemistry (IHC) showed negative. Obviously, the tumor was considered unresectable. We did plasma gene detection for this patient. As a result, plasma BRCA1 was low expressed (1.27, following the cut-off of Table 3), TS was high expressed (1.06, following the cut-off of table 6) while TOPO1 was low expressed (0.25, following the cut-off of Table 3). Therefore, the chemo strategy for this patient was Oxaliplatin plus S-1(Oxaliplatin 85mg/m², VD, d1,15, S-1 40mg/m², bid, po, d1-14, q28d). As shown by CT scan, there was partial remission after 2 cycles of chemotherapy with diameter of the biggest one from $9.6 \mathrm{~cm}$ to $3.5 \mathrm{~cm} .4$ cycles later, the disease as well controlled. 6 cycles later, GI endoscopy showed complete remission of tumor in stomach; while CT scan showed metastatic tumor in liver was stable with the longest diameter of $2.6 \mathrm{~cm}$. Then radiation therapy was given on the tumor of liver. After radiation, the patient was followed up and was still in good condition now.
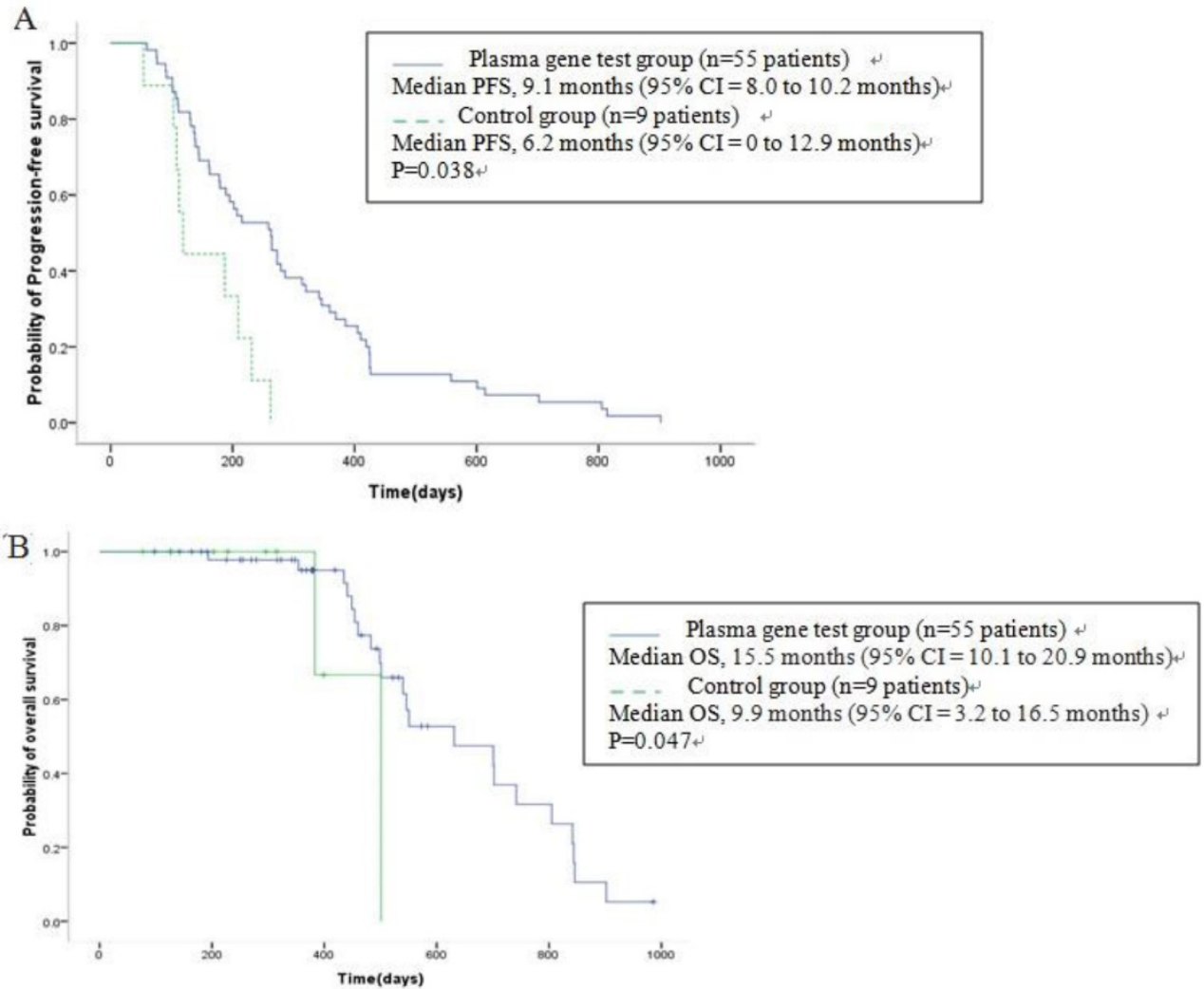

Figure 4. Kaplan-Meier estimates of progression-free-survival (A) and overall survival (B) in advanced (stage IV) gastric cancer patients. A total of 55 patients received chemotherapy according to their plasma gene detection (see Table 4), and another 9 were treated with docetaxel+S-1 as control. OS = overall survival; PFS: progression-free-survival; $\mathrm{Cl}=$ confidence interval. 

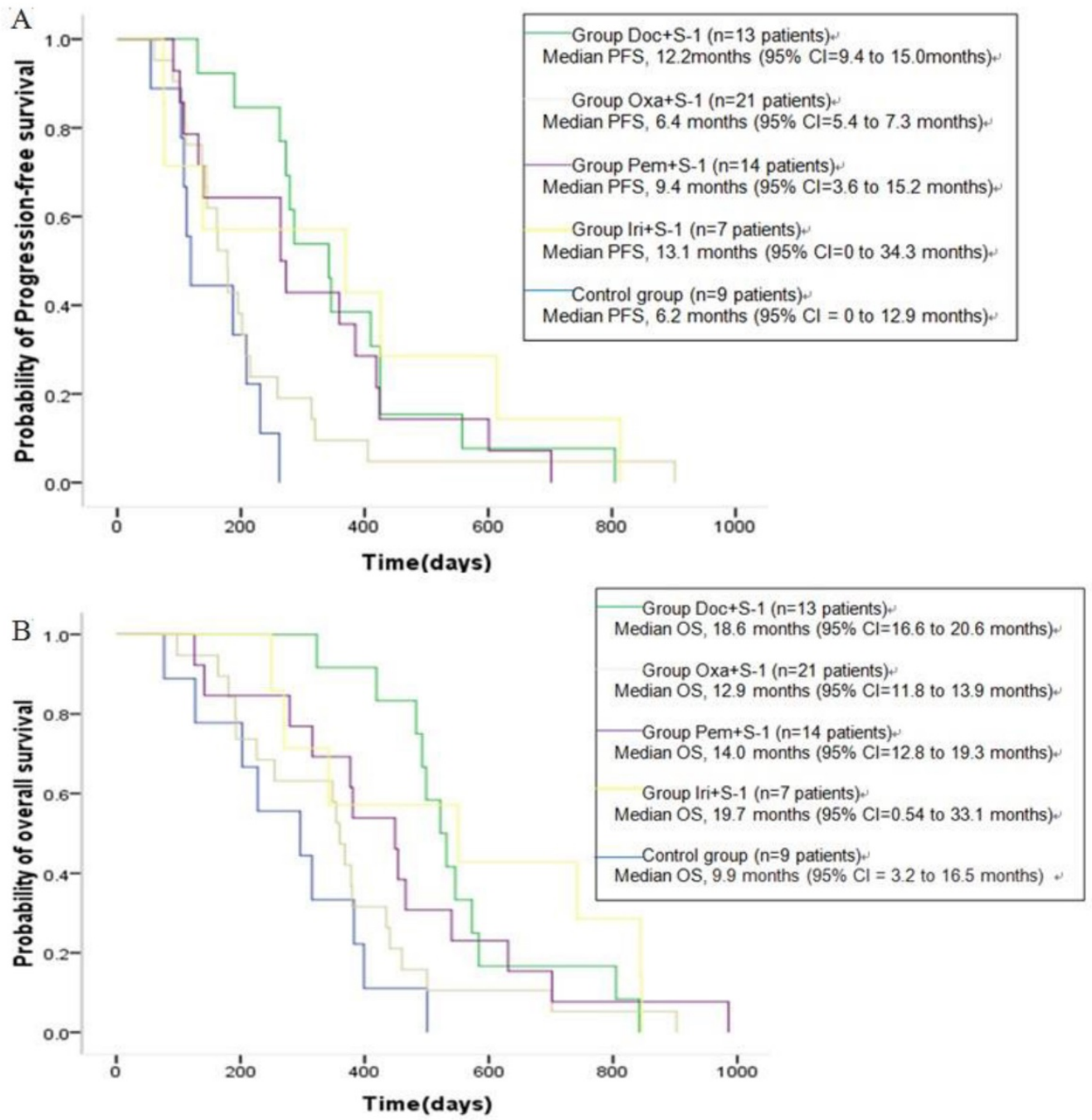

Figure 5. PFS(A) and OS (B) of different groups of individualized chemotherapy and control.

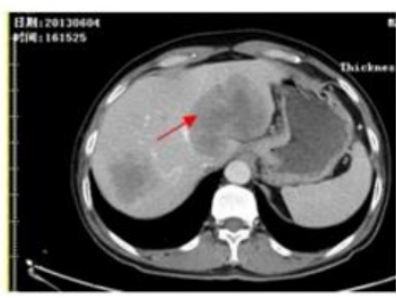

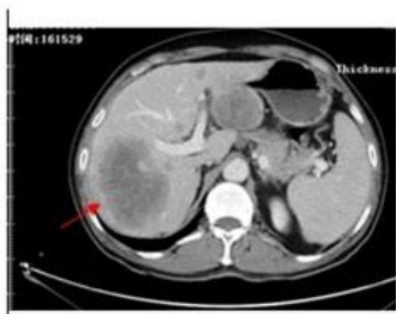

Before chemo (2013-06-04)

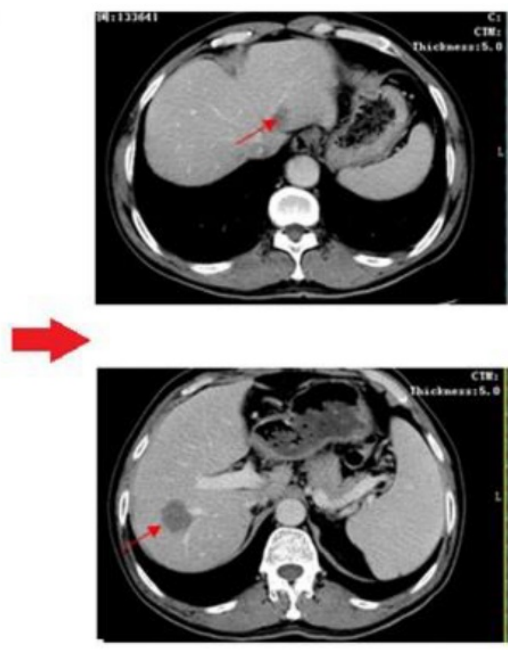

2 cycles after chemo (2013-08-23)

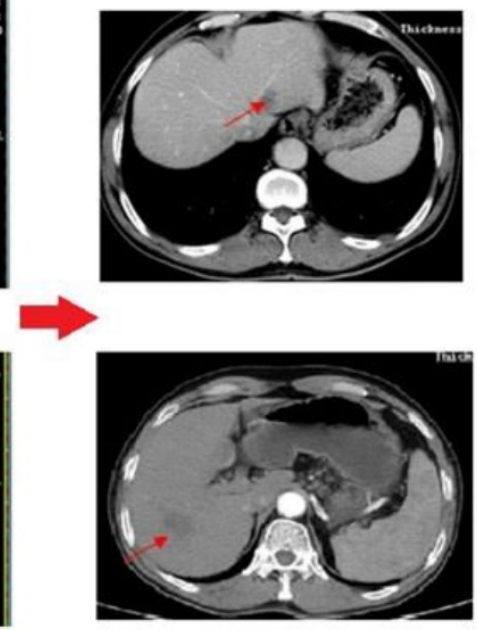

4 cycles after chemo (2013-11-25).

Figure 6. The CT scan of the patient received chemotherapy according to his plasma gene detection. There was partial remission after 2 cycles of chemotherapy. 4 cycles later, the disease was well controlled. 
Table 7. Median overall survival and hazard ratios for risk of mortality in 64 stage IV gastric cancer patients receiving control or individualized chemotherapy.

\begin{tabular}{|c|c|c|c|c|c|}
\hline \multirow[t]{2}{*}{ Variable } & \multirow{2}{*}{$\begin{array}{l}\text { No. of } \\
\text { patients }\end{array}$} & \multicolumn{2}{|l|}{ Overall survival } & \multicolumn{2}{|l|}{ OS of mortality } \\
\hline & & Month(95\% CI) & $\mathrm{P}$ & $\mathrm{HR}(95 \% \mathrm{CI})$ & $\mathrm{P}$ \\
\hline Palliative & & & 0.855 & & \\
\hline \multicolumn{6}{|l|}{ Surgery } \\
\hline No & 47 & $12.7(9.4$ to 16.0$)$ & & $1.23(0.48$ to 3.20$)$ & 0.668 \\
\hline Yes & 17 & $14.0(8.0$ to 20.0$)$ & & 1.00 (referent) & \\
\hline ECOG PS & & & 0.003 & & \\
\hline $0-1$ & 48 & $16.4(11.4$ to 21.4$)$ & & 1.00 (referent) & \\
\hline$>=2$ & 16 & 10.5 (7.3 to 13.7$)$ & & $2.55(1.12$ to 5.83$)$ & 0.026 \\
\hline Age & & & 0.418 & & \\
\hline$<60 y$ & 25 & $17.4(9.3$ to 25.5$)$ & & 1.00 (referent) & \\
\hline$>=60 y$ & 39 & $12.6(11.2$ to 14.0$)$ & & $1.37(0.66$ to 2.85$)$ & 0.401 \\
\hline $\begin{array}{l}\text { Histological } \\
\text { grade }\end{array}$ & & & 0.762 & & \\
\hline 2 & 34 & $13.3(9.0$ to 17.6$)$ & & $0.96(0.33$ to 2.84$)$ & 0.946 \\
\hline 3 & 16 & $12.3(8.4$ to 16.1$)$ & & $1.57(0.46$ to 5.36$)$ & 0.470 \\
\hline unknown & 14 & $14.0(6.6$ to 21.4$)$ & & 1.00 (referent) & \\
\hline $\begin{array}{l}\text { Metastasis No.of } \\
\text { sites involved }\end{array}$ & & & 0.006 & & \\
\hline 1 & 27 & $19.1(15.6$ to 22.6$)$ & & 1.00 (referent) & \\
\hline$>=2$ & 28 & 10.8 (8.9 to 12.6$)$ & & $2.16(1.01$ to 4.65$)$ & 0.048 \\
\hline \multicolumn{6}{|l|}{ Chemotherapy } \\
\hline Control & 9 & $9.9(3.2$ to 16.5$)$ & 0.047 & $2.34(0.93$ to 5.88$)$ & 0.071 \\
\hline Plasma gene test & 55 & $15.5(10.1$ to 20.9$)$ & & 1.00 (referent) & \\
\hline
\end{tabular}

\section{Discussion}

In the present study, we demonstrated the value of plasma mRNA as predictive biomarker for individualized chemotherapy. We firstly proved that BRCA1, TS, and TOPO1 mRNA levels tested in both tumor and plasma have relationship with in vitro chemosensitivity of fresh tumor tissues. Then, individualized chemotherapy based on plasma mRNA levels could bring longer survival and progress free time for advanced gastric patients. These findings suggested that plasma mRNA as liquid biopsy could be ideal recourse for examination to predict chemo-sensitivity in gastric cancer.

As we all know, platinum (cisplatin or oxaliplatin) combined with other agents, such as 5-FU, docetaxel, and irinotecan were recommended as first-line chemo-agents for advanced gastric cancer patients[1]; even though, the median survival remains one year. S-1, an oral anticancer drug with the advantage of high concentrations of fluorouracil in serum and tumor, has been recommended as first line for advanced gastric cancer [20]. No standard treatment method was recommended for second-line therapy, although some agents show some activity [2]. Therefore, it is reasonable and necessary to screen and validate useful biomarkers to select best treatment for advanced gastric cancer patients.

In a phase III trial, it is reported that by assigning to cisplatin plus S-1, the media OS of advanced gastric patients could reach $13.0 \mathrm{~m}$ and PFS could reach $6.0 \mathrm{~m}$ [20]. Another phase III trial showed that OS was $14.1 \mathrm{~m}$ and PFS reached $5.5 \mathrm{~m}$ for Oxaliplatin plus S-1, while OS was $13.1 \mathrm{~m}$ and PFS reached $5.4 \mathrm{~m}$ for cisplatin plus S-1[21].It also has been reported in a phase II trial that when irinotecan plus S-1 were given for advanced gastric cancer patients, the OS were 12.4 months and PFS were 5.7 months; while when paclitaxel plus S-1 were given, the OS were 11.9 months and PFS were 4.6 months, respectively[22]. In ToGA trial, it is reported that by assigning to trastuzumab plus chemotherapy, the median OS of advanced gastric patients could reach $13.8 \mathrm{~m} \mathrm{(95 \%} \mathrm{CI}$ 12-16) compared with $11.1 \mathrm{~m}$ (95\% CI 10-13) in patients assigned to chemo alone, while PFS was 6.7 months vs. 5.5 months[23]. Therefore, from the clinical evidences mentioned above, we understand that the longest OS for advanced gastric cancer was approximately 14.1 months and PFS was 6.7 months even trastuzumab were used. Then, fortunately, based on plasma gene detection, we could improve OS for advanced gastric cancer patients from 14.1 months to 15.5 months, and PFS from 6.7 months to 9.1 months. The plasma based individualized chemotherapy could bring benefit to advanced gastric cancer, especially in improving PFS. Blood could be a useful and non-invasive tool to provide real-time information in the procedure of treatment.

Plasma mRNA was thought to be from tumor or apoptosis cells [8]. It was demonstrated to be stable in blood $[8,14]$. Although some cell-free mRNA has been reported recently [24-27], the aim of all those studies was focused on prognostic biomarkers, which are used for diagnosis and tumor monitoring. In our previous work, we have carried out primary investigation of plasma mRNA levels of BRCA1 and TS; however, that work is in vitro test based. Besides that, there is no publications investigating the potential role of plasma mRNA to predict chemo-efficiency in any solid cancers including gastric cancer. The present study fills the gap, and provides useful evidence for the role of plasma mRNA as liquid biopsy in personalized treatment practices.

In conclusion, the current study has indicated an important role of plasma mRNA as liquid biopsy to provide useful predictive information in gastric cancer treatment, especially for those who need re-biopsy when clinical progression appears or chemo-scheme need to be made when there were not enough samples for gene testing. However, we have to admit that there were some shortages of the current study. One was that there were not enough patients in each subgroup, and the other was that we did not compare the plasma gene levels with those expressed 
of tumors in validate study. The main reason of those defects was that there were not enough tumor samples for gene detection when diagnosed biopsy was made for stage IV patients. To overcome the shortage above, a large-scaled clinical trial (ChiCTR-IPR-15006201) has been designed, registered and carried out in our cancer center to further validate the value and clinical potential of plasma mRNA (BRCA1, TS, and TOPO1) based individualized chemotherapy in advanced gastric cancer.

\section{Acknowledgement}

This work was funded by grants from the National Natural Science Foundation of China (Grant No. 81401969, 812101060 and 81300339) and the Natural Science Foundation of Jiangsu Province (Grant No. BK20130092). The sponsors of the study had no role in study design, data collection, data analysis, data interpretation, or writing of the report. The corresponding author had full access to all the data in the study and had final responsibility for the decision to submit for publication.

\section{Authors' contributions}

JS, BRL and JW are responsible for the study designing and experiment adjustment. JS, YNW, LXY and $Y Y$ performed the experiments involved, draft the manuscript, and conducted statistical analysis. WWK, HZR, YY and WXG collected the blood sample and clinical information of patients. All authors read and approved the final manuscript.

\section{Competing interests} interests.

The authors declare that they have no competing

\section{References}

1. Cunningham D, Starling N, Rao S, et al. Capecitabine and oxaliplatin for advanced esophagogastric cancer. N Engl J Med. 2008; 358(1): 36-46.

2. Wesolowski R, Lee C, Kim R. Is there a role for second-line chemotherapy in advanced gastric cancer? Lancet Oncol. 2009; 10(9): 903-12.

3. Quinn JE, Kennedy RD, Mullan PB, et al. BRCA1 functions as a differential modulator of chemotherapy-induced apoptosis. Cancer Res. 2003; 63(19): 6221-8.

4. Quinn JE, James CR, Stewart GE, et al. BRCA1 mRNA expression levels predict for overall survival in ovarian cancer after chemotherapy. Clin Cancer Res. 2007; 13(24): 7413-20.

5. Wang L, Wei J, Qian X, et al. ERCC1 and BRCA1 mRNA expression levels in metastatic malignant effusions is associated with chemosensitivity to cisplatin and/or docetaxel. BMC Cancer. 2008; 8: 97.

6. Wei J, Costa C, Ding Y, et al. mRNA expression of BRCA1, PIAS1, and PIAS4 and survival after second-line docetaxel in advanced gastric cancer. J Natl Cancer Inst. 2011; 103(20): 1552-6.

7. Monaco SE, Nikiforova MN, Cieply K, et al. A comparison of EGFR and KRAS status in primary lung carcinoma and matched metastases. Hum Pathol. 2010; 41(1): 94-102.

8. Schwarzenbach H, Hoon DS, Pantel K. Cell-free nucleic acids as biomarkers in cancer patients. Nat Rev Cancer. 2011; 11(6): 426-37.

9. Baldus SE, Schaefer KL, Engers R, et al. Prevalence and heterogeneity of KRAS, BRAF, and PIK3CA mutations in primary colorectal adenocarcinomas and their corresponding metastases. Clin Cancer Res. 2010; 16(3): 790-9.

10. Kalikaki A, Koutsopoulos A, Trypaki M, et al. Comparison of EGFR and K-RAS gene status between primary tumours and corresponding metastases in NSCLC. Br J Cancer. 2008; 99(6): 923-9.
11. Badalian $\mathrm{G}$, Barbai $\mathrm{T}$, Raso $\mathrm{E}$, et al. Phenotype of bone metastases of non-small cell lung cancer: epidermal growth factor receptor expression and K-RAS mutational status. Pathol Oncol Res. 2007; 13(2): 99-104.

12. Salazar F, Molina MA, Sanchez-Ronco $M$, et al. First-line therapy and methylation status of CHFR in serum influence outcome to chemotherapy versus EGFR tyrosine kinase inhibitors as second-line therapy in stage IV non-small-cell lung cancer patients. Lung Cancer. 2011; 72(1): 84-91.

13. Nabeya Y, Suzuki T, Furuya A, et al. Calpain regulates thymidylate synthase-5-fluoro-dUMP complex levels associated with response to 5-fluorouracil in gastric cancer cells. Cancer Sci. 2011; 102(8): 1509-15.

14. Garcia JM, Garcia V, Pena C, et al. Extracellular plasma RNA from colon cancer patients is confined in a vesicle-like structure and is mRNA-enriched. RNA. 2008; 14(7): 1424-32.

15. Shen J, Wang $\mathrm{H}$, Wei J, et al. Thymidylate synthase mRNA levels in plasma and tumor as potential predictive biomarkers for raltitrexed sensitivity in gastric cancer. International Journal of Cancer. 2012; 131(6): E938-45.

16. Shen J, Wei J, Guan W, et al. Plasma mRNA expression levels of BRCA1 and TS as potential predictive biomarkers for chemotherapy in gastric cancer. J Transl Med. 2014; 12(1):355.

17. Cecere F, Bria E, Rosell R. DNA repair by ERCC1 in non-small-cell lung cancer. N Engl J Med. 2006; 355(24): 2590-1.

18. Shen J, Wei J, Wang $\mathrm{H}$, et al. A three-gene signature as potential predictive biomarker for irinotecan sensitivity in gastric cancer. J Transl Med. 2013; 11:73.

19. Dopeso $\mathrm{H}$, Mateo-Lozano $\mathrm{S}$, Elez E, et al. Aprataxin tumor levels predict response of colorectal cancer patients to irinotecan-based treatment. Clin Cancer Res. 2010; 16(8): 2375-82.

20. Koizumi W, Narahara H, Hara T, et al. S-1 plus cisplatin versus S-1 alone for first-line treatment of advanced gastric cancer (SPIRITS trial): a phase III trial. Lancet Oncol. 2008; 9: 215-21.

21. Yamada $Y$, Higuchi K, Nishikawa $K$, et al. Phase III study comparing oxaliplatin plus S-1 with cisplatin plus S-1 in chemotherapy-naïve patients with advanced gastric cancer. Annals of Oncology. 2015; 26: 141-8.

22. Sugimoto N , Fujitani K, Imamura H, et al. Randomized Phase II Trial of S-1 plus Irinotecan Versus S-1 plus Paclitaxel as First-line Treatment for Advanced Gastric Cancer (OGSG0402). Anticancer research. 2014; 34: 851-8.

23. Bang YJ, Van Cutsem E, Feyereislova A, et al. Trastuzumab in combination with chemotherapy versus chemotherapy alone for treatment of HER2-positive advanced gastric or gastro-oesophageal junction cancer (ToGA): a phase 3, open-label, randomised controlled trial. Lancet. 2010; 376: 687-97.

24. Silva JM, Rodriguez R, Garcia JM, et al. Detection of epithelial tumour RNA in the plasma of colon cancer patients is associated with advanced stages and circulating tumour cells. Gut. 2002; 50(4): 530-4.

25. Wong SC, Lo SF, Cheung MT, et al. Quantification of plasma beta-catenin mRNA in colorectal cancer and adenoma patients. Clin Cancer Res. 2004; 10(5): 1613-7.

26. Terrin L, Rampazzo E, Pucciarelli S, et al. Relationship between tumor and plasma levels of hTERT mRNA in patients with colorectal cancer: implications for monitoring of neoplastic disease. Clin Cancer Res. 2008; 14(22): 7444-51.

27. Garcia V, Garcia JM, Pena C, et al. Free circulating mRNA in plasma from breast cancer patients and clinical outcome. Cancer Lett. 2008; 263(2): 312-20. 